\title{
A NOTE ON FINITE GROUPS IN WHICH NORMALITY IS TRANSITIVE
}

\author{
DEREK J. S. ROBINSON
}

1. Introduction. We will say that a group $G$ satisfies the condition $\mathfrak{e}_{p}$ (where $p$ is a prime) if every subgroup of a Sylow $p$-subgroup $P$ of $G$ is normal in the normalizer of $P$. Here we wish to consider the relation between the condition $\mathfrak{C}_{p}$ and the class $\mathfrak{J}$ of all groups in which normality is a transitive relation. More precisely $G \in J$ if and only if $H \triangleleft K \triangleleft G$ always implies that $H \triangleleft G$. Our object here is to prove

TheOREM 1. A finite group which satisfies $\mathfrak{C}_{p}$ for all $p$ is a soluble J-group.

Let $\overline{\mathfrak{J}}$ denote the class of all groups $G$ for which $H \triangleleft K \triangleleft L \leqq G$ always implies that $H \triangleleft L$ : in short $\overline{\mathfrak{J}}$ is the largest subclass of $\mathfrak{J}$ that is closed with respect to forming subgroups. Now every finite soluble $\sqrt{5}$-group is a $\overline{\mathfrak{J}}$-group $[2 \text {, Satz } 4]^{1}$ and it is obvious that a finite $\overline{\mathfrak{J}}$-group satisfies $\mathfrak{C}_{p}$ for all $p$, since every subgroup of a finite $p$-group is subnormal. Consequently we have

THEOREM 1*. If $G$ is a finite group, the following are equivalent statements.

(i) $G$ is soluble J-group.

(ii) $G$ is a $\bar{J}$-group.

(iii) $G$ satisfies $\mathfrak{e}_{p}$ for all $p$.

Every soluble J-group is metabelian [4, Theorem 2.3.1], so Theorem $1^{*}$ yields the following information about infinite $\overline{\mathfrak{J}}$-groups.

Corollary. A locally finite $\overline{\mathrm{J}}$-group is soluble.

The proof of Theorem 1 uses the Schur-Zassenhaus splitting theorem, Burnside's theorem on the existence of a normal complement of a Sylow subgroup that lies in the centre of its normalizer and Grün's First Theorem [3]. In addition we need some simple facts about $J$ groups, the first of which has already been mentioned.

(A) Soluble J-groups are metabelian.

(B) Let $N \triangleleft G$ where every subnormal subgroup of $N$ is normal in $G, G / N$ belongs to $\mathrm{I}$ and the order of $N$ is prime to its index. Then $G$ belongs to $J[4$, Lemma 5.2.2].

Received by the editors March 22, 1967.

${ }^{1}$ However, not every finite $\mathfrak{J}$-group or infinite soluble $\mathfrak{J}$-group is in $\bar{J},[4]$. 
(C) If $A$ is a finite abelian group and $\alpha$ is a power automorphism of $A$ (i.e. an automorphism which leaves every subgroup of $A$ invariant), then there exists a positive integer $m$ such that $a^{\alpha}=a^{m}$ for all $a \in A,[2$, p. 88].

2. $\mathfrak{e}_{p}$ and $p$-nilpotence. We will prove Theorem 1 via two preliminary results connecting $\mathfrak{C}_{p}$ with the notion of $p$-nilpotence. (Recall that a finite group $G$ is $p$-nilpotent if it has a normal subgroup of index a power of $p$ and of order prime to $p$.)

THEOREM 2. If the finite group $G$ satisfies $\mathfrak{C}_{p}$ where $p$ is the smallest prime dividing the order of $G$, then $G$ is p-nilpotent.

Proof. Let $P$ be a Sylow $p$-subgroup of $G$ and let $N=N_{G}(P)$, its normalizer in $G$. Every subgroup of $P$ is normal in $N$, so $P$ is either abelian or Hamiltonian. Suppose that $p$ is odd, so that $P$ is abelian. Elements of $N$ induce power automorphisms in $P$ of order prime to $p$ and therefore dividing $p-1$. Since $p$ is the smallest prime dividing the order of $G, P$ lies in the centre of $N$ and by Burnside's theorem $P$ has a normal complement, i.e. $G$ is $p$-nilpotent. Now let $p=2$. By the Schur-Zassenhaus theorem $N$ splits over $P$ and so we may write $N=P H$, with $P \cap H=1$. A power automorphism of $P$ has order a power of 2 (whether or not $P$ is abelian) and $H$ has odd order. Hence $H$ centralizes $P$ and $N=P \times H$.

If $P$ is abelian, it is central in $N$ and we can again use Burnside's theorem. Suppose therefore that $P$ is Hamiltonian.

Let $\sigma$ denote the transfer of $G$ in to $P / P^{\prime}$ and let $K=\operatorname{Ker} \sigma$. Then

$$
G / K \cong P / P^{*}
$$

where

$$
P^{*}=\left(P \cap N^{\prime}\right) \prod_{g \in G}\left(P \cap\left(P^{\prime}\right)^{g}\right)
$$

by Grün's First Theorem. $P \cap\left(P^{\prime}\right)^{g}$ and $\left(P \cap\left(P^{\prime}\right)^{g}\right)^{g^{-1}}$ are both normal in $P$ and hence by a standard "Sylow" argument [3, Lemma 14.3.1] these subgroups are conjugate in $N$. By $\mathfrak{C}_{p}$

$$
P \cap\left(P^{\prime}\right)^{g}=\left(P \cap\left(P^{\prime}\right)^{g}\right)^{\sigma^{-1}}
$$

and therefore $P \cap\left(P^{\prime}\right)^{g} \leqq P^{\prime}$ for all $g \in G$. On the other hand since $N=P \times H, P \cap N^{\prime}=P^{\prime}$. Thus we conclude that $P^{*}=P^{\prime}$, which by the structure of Hamiltonian groups [3, Theorem 12.5.4] has order 2. Since $G / K \cong P / P^{\prime}, P^{\prime}$ is a Sylow 2-subgroup of $K$ and clearly it must lie in the centre of $N_{K}\left(P^{\prime}\right)$. Therefore $P^{\prime}$ has a normal complement in $K$ 
and this is obviously also a normal complement for $P$ in $G$. This completes the proof.

The solubility of groups of odd order yields the following

Corollary. A finite group satisfying $\mathfrak{C}_{2}$ is soluble.

The alternating group of degree 5 satisfies $\mathfrak{C}_{3}$ and $\mathfrak{C}_{5}$ but not $\mathfrak{C}_{2}$, so the hypothesis that $p$ is "smallest" cannot be omitted from the statement of Theorem 2. Let us say that a group is p-perfect if it has no nontrivial abelian $p$-factor groups. A finite group that is both $p$-nilpotent and $p$-perfect has order prime to $p$, so that these properties represent extremes of behaviour for finite groups. It is therefore interesting that the condition $\mathfrak{e}_{p}$ forces a finite group to one of those extremes. This is

Theorem $3 .{ }^{2}$ If the finite group $G$ satisfies $\mathfrak{C}_{p}, G$ is either p-nilpotent or p-perfect.

Proof. Let $P$ be a Sylow $p$-subgroup of $G$ and let $N=N_{G}(P)$. If $P$ lies in the centre of $N, G$ is $p$-nilpotent, so we can suppose that $[P, x] \neq 1$ for some $x \in N$. In addition we may assume that $p>2$, otherwise $G$ would again be $p$-nilpotent, by Theorem 2 . Hence $P$ is abelian and by (C) there is an $m>0$ such that $a^{x}=a^{m}$ for any $a \in P$. If $m \equiv 1 \bmod p, m^{p^{i}} \equiv 1 \bmod p^{i+1}$ for $i \geqq 0$, and the automorphism induced in $P$ by $x$ would have order a power of $p$; since $|N: P|$ is prime to $p$, this automorphism would have to be trivial. Hence $m \neq 1 \bmod p$ and so

$$
\langle[a, x]\rangle=\left\langle a^{m-1}\right\rangle=\langle a\rangle
$$

for all $a \in P$. Consequently $P \leqq G^{\prime}$, which shows that $G$ is $p$-perfect.

Proof of Theorem 1. Suppose that $G$ is a finite group of least order such that $G$ satisfies $\mathcal{C}_{p}$ for all $p$ and yet $G$ is not a soluble 3-group. Let $p$ be the smallest prime dividing the order of $G$, so that $G$ is $p$-nilpotent by Theorem 2 . We can write $G=P H$ where $H \triangleleft G$, $H \cap P=1$ and $P$ is a Sylow $p$-subgroup of $G$. The order of $H$ is prime to its index in $G$, so $H$ satisfies all the conditions $\mathfrak{C}$ and is a soluble J-group by minimality of $G$; thus $G$ is certainly soluble and to obtain a contradiction we have only to show that $G$ belongs to J. Suppose that $H$ is nilpotent and hence abelian (being of odd order). Let $q$ be a prime dividing the order of $H$; then $Q$, the $q$-primary component of $H$, is the unique Sylow $q$-subgroup of $G$ and hence each subgroup of $Q$ is normal in $G$, by $\mathfrak{e}_{q}$. $G$ splits over $Q$ with, say, $G=K Q$ and $K \cap Q$

2 See $[6$, p. 59] for a special case of this result. 
$=1 ; K$ satisfies all the conditions $\mathbb{C}$ and is therefore a $J$-group, so that $G / Q$ is a I-group. That $G$ belongs to I now follows by (B). Next we suppose that $H$ is non-nilpotent, so there is a prime $q$ dividing the order of $H$ such that $H$ is not $q$-nilpotent. By (A) $H^{\prime}$ is abelian and Theorem 3 shows that $H$ is $q$-perfect and so $H / H^{\prime}$ has order prime to $q$. Hence $Q$, the $q$-primary component of $H^{\prime}$, is the unique Sylow $q$-subgroup of $G$ and has each of its subgroups normal in $G$. $G$ splits over $Q$ and $G / Q$ belongs to $J$ by minimality. Finally $G$ belongs to $J$ by (B) as before.

3. Pronormal subgroups. A subgroup $H$ of a group $G$ is said to be pronormal in $G$ if for any $g \in G, H$ and $H^{g}$ are already conjugate in their join $\left\langle H, H^{o}\right\rangle$. There is a link between pronormality and the condition $\mathfrak{C}_{p}$ which was pointed out by Dr. J. S. Rose (to whom the author is indebted for several useful comments).

Lemma (J. S. Rose). A finite group $G$ satisfies $\mathfrak{C}_{p}$ if and only if every p-subgroup is pronormal in $G$.

Proof. Assume that $G$ satisfies $\mathcal{C}_{p}$ and let $P_{0}$ be any $p$-subgroup of $G$. Let $g \in G$; we show that $P_{0}$ and $P_{0}^{o}$ are conjugate in $J=\left\langle P_{0}, P_{0}^{o}\right\rangle$. Let $P_{1}$ be a Sylow $p$-subgroup of $J$ containing $P_{0}$. Then for some $x \in J, P_{0}^{o} \leqq P_{1}^{x}$; hence $P_{0}$ and $P_{0}^{o x^{-1}}$ are both contained in $P_{1}$. Let $P$ be a Sylow $p$-subgroup of $G$ containing $P_{1}$. By $\mathcal{C}_{p} P_{0}$ and $P^{o x^{-1}}$ are both normal in $P$ and hence are conjugate in $N_{G}(P)$; by $\mathfrak{C}_{p}$ again, $P_{0}$ $=P_{0}^{x^{-1}}$ and so $P_{0}^{o}=P_{0}^{x}$. To prove the converse note that pronormality and subnormality together imply normality.

Corollary. For finite groups the condition $\mathfrak{e}_{p}$ is inherited by subgroups and homomorphic images.

Proof. The subgroup part is clear. Let $H / N$ be a $p$-subgroup of $G / N$ where $G$ satisfies $\mathcal{C}_{p}$ and let $P$ be a Sylow $p$-subgroup of $H$. By comparison of orders $H=P N . P$ is pronormal in $G$ and clearly this implies that $H / N$ is pronormal in $G / N$. It will be noted that in the proof of Theorem 1 the fact that $\mathfrak{C}_{p}$ passes to subgroups was used only in situations where this was obvious. ${ }^{3}$ Observe also that the main theorem can be formulated thus:

a finite group in which for each p every cyclic p-subgroup is pronormal is a soluble J-group. On the other hand it is known that in a soluble J-group all subgroups are pronormal [5].

${ }^{3}$ This property may be combined with the well-known theorem of Frobenius on $p$-nilpotent groups [7, Theorem iv. 5.c] to give another proof of Theorem 2. 
The above corollary allows us to draw a further conclusion from Theorem 3.

Corollary (To Theorem 3). A finite group which satisfies $\mathfrak{e}_{p}$ and is p-soluble has p-length $\leqq 1$.

For if $G$ is such a group every subgroup of $G$ is either $p$-nilpotent or $p$-perfect and this excludes the possibility of a subgroup of $p$ length 2. (This result also follows from the main theorem of [1].)

On the other hand such a group need not be $p$-nilpotent, as the symmetric group of degree 4 with $p=3$ shows.

\section{REFERENCES}

1. S. Bauman, p-normality and p-length of a finite group, Math. Z. 87 (1965), 345347.

2. W. Gaschütz, Gruppen in denen das Normalteilersein transitiv ist, J. Reine Angew. Math. 198 (1957), 87-92.

3. M. Hall, The theory of groups, Macmillan, New York, 1959.

4. D. J. S. Robinson, Groups in which normality is a transitive relation, Proc. Cambridge Philos. Soc. 60 (1964), 21-38.

5. J. S. Rose, The abnormal structure of finite groups, Ph.D. Dissertation, Cambridge Univ., 1964.

6. - On a splitting theorem of Gaschütz, Proc. Edinburgh Math. Soc. (2) 15 (1966), 57-60.

7. E. Schenkman, Group theory, Van Nostrand, Princeton, N. J., 1965.

Queen Mary College, London, England 